\title{
Test Time Reduction in Scan Designed Circuits
}

\author{
Wen-Joung Lai, Chen-Pin Kung \& Chen-Shang Lin \\ Department of Electrical Engineering \\ National Taiwan University \\ Taipei, Taiwan, R.O.C.
}

\begin{abstract}
The reduction of test application time for the general scan designed circuits had been studied. The reduction problem had been investigated from three aspects. the test generation, selective scan, and rearrangement of scan path. The two phase testing strategy had been proposed to employ scan only for the hard-to-detect faults. Four cases of selective scan had also been $i$ dentified. Furthermore, an ordering heuristic without layout constraint had been proposeded to maximize the reduction of unnecessary scans and hence the test application time. Applying these reduction methods, the total test clock-cycles can be reduced to only $20 \%$ on average for ISCAS sequential benchmark circuits with partial scan.
\end{abstract}

\section{Introduction}

The progress of vary large scale integration(VLSI) technology has brought tremendous improvement in chip performance as well as gate density. The increasing complexity, however, renders the testing of a VLSI chip much more difficult. For large sequential circuits, the automatic test pattern generation (ATPG) is quite time consuming and the resultant fault coverage is often unacceptably low. The remedy is naturally to take into account the testability while designing a chip, i.e., the design for testability. The most popular approach in design for testability is the scan design such as full scan[1] and partial scan[2]. In the scan design, all or part of flip-flops(FFs) in a chip are interconnected into a scan chain during test mode so that all these scan flip-flops(SFFs) can be controlled/observed by scanin/scan-out operations. As a result, the test generation problem can be significantly reduced[4]. However, the test application time of a scan-designed circuit is in general lengthened because of the extensive scan operations to shift in the patterns and shift out the responses. The reduction of test application time for scan designed circuits is the main concern of this paper.

To reduce the scan operations in a scan designed circuit, a straightforward way is to increase the number of parallel scan chains, with additional overhead in pins. Recently, novel approaches $[5,6,7]$ have been

tThis work was supported in part by the National Science Council R.O.C under Grant NSC 82-0404-E-002-167. proposed to reduce test application time by rearranging and modifying the scan path. These approaches are constrained by the given test sequence and do not exploit the benefit from test generation. Moreover, some $[6,7]$ even have relied on the pattern independence of the full-scan circuits, which in general does not hold in the partial-scan designed circuits.

In this paper, the reduction of test application time of general scan designed circuits is studied. The problem is investigated from three aspects: the test generation, selective scan, and rearrangement of scan path. It will be shown in our experimental result that by allowing arbitrarily ordering of scanned flip-flops in the partial scan chain, the test application time can be reduced to only $20 \%$ on average.

In the next section, the reduction methods for the test application time of scan designed circuits will be described. Then the evaluation results on the sequential benchmark circuits will be provided. And finally the conclusion will be given.

\section{Test Time Reduction for Scan De-} signed Circuits

In this section, our reduction method for the test application time of scan designed circuits will be described.

First we will introduce a measure of test application time. In a scan designed circuit, the content of the scanned flip-flops (SFFs) can be controlled and observed by serial shift operations of the scan chains. Let $S$ be the number of scanned FFs, $T$ the number of test patterns, and $P$ the number of scan chains. Then for each applied test pattern, $S / P$ shift operations plus one actual testing operation are required. The response to be shifted out can be overlapped with the scan-in of the next pattern. Assuming that both shift operation and testing operation take the same amount of time, say one clock cycle, then for each pattern, $(S / P+1)$ clock cycles are required and for $T$ patterns, $T *(S / P+1)+S / P$ where the last term $S / P$ is due to the response scan-out of the last pattern. Define the above required clock-cycles as the total test clock-cycles (TTCs), i.e.,

$$
T T C s=T *(S / P+1)+S / P .
$$

Without loss of generality, we assume that there is only one scan chain in the given circuit, $P=1$. 
Given a scan path, the test application time can be reduced by exploiting and eliminating unnecessary scan operations. The reduction can be achieved in the test generation, selective scan, and the arrangement of scan path. They will be briefly described in the sequel.

\subsection{Two Phase Test Generation}

The two phase test generation strategy is based on the observation that in most scan designed circuits, a majority of faults can be easily detected even without scan. To take advantage of the above observation for reducing scan, the testing of a circuit can be separated into two phases: in the first phase, the circuit is tested as a scanless circuit, and only in the second phase is the scan path utilized. To accommodate this testing strategy, the test generation is also split into two phases: the scanless phase for easy-to-detect faults and the scan phase for hard-to-detect faults.

One might suspect that by splitting into two phases, the the test application time would be adversely lengthened. It will be demonstrated later that, with careful consideration, the converse is true. Significan$t$ reduction in scan and test application time can be obtained with this simple two phase testing strategy.

The balance between these two phases can be determined as follows. In a sequential ATPG, the limit of iterative time frames $(F)$ can be set by users. A fault which can be test generated within $F$ is in phase 1 , otherwise it belongs to phase 2 . Clearly, too large an $F$ would include many hard-to-detect faults in the first phase and inevitably lengthen the test sequence. On the other hand, a small $F$ could drive most faults to the second phase and thus need more scan operations. Since the test cycles to place a given fault in phase 1 is no more than $F$ and in phase 2 is proportional to $(S+1)$, which is the test cycle number for each test pattern, we could restricted iterative time frames in phase 1 such that $F<K *(S+1)$, where $K$ is a given number. The implication of $K$ is the average number of test patterns for each fault to be detected in phase 2 . The number $K$ unfortunately can not be known a priori. However, in our experiment, it is found that $K=2$ consistently quite good result in all evaluated cases. A slightly better result is obtained by simply letting our ATPG determine when to abort a fault to phase 2 .

It is also worth noting that the two phase testing strategy does not require any additional hardware, i.e., no overhead except that of an ordinary partial scan.

\subsection{Selective Scan}

The objective of shift operations in the scan design is to control the scan elements and to observe the effect of the previous pattern. However, it is not always necessary to control and observe every scanned element in every test pattern. Certain bits in a given pattern may be don't-care and response bits provide detection information only occasionally[5]. This sparseness of information in SFFs can be further explored during the test generation.

During the second phase of the test generation, we modify the ATPG such that it deliberately leaves the pseudo PIs (SFFs) in don't-care whenever possible.

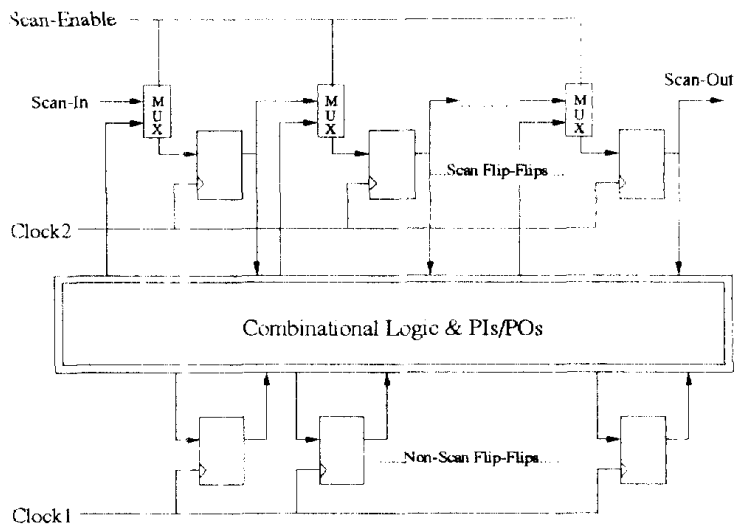

Figure 1: Scan Facility

The results show that averagely less than $28 \%$ of the scanned FFs in a circuit are used for actual test applying purpose, and less than $8 \%$ for actual response observing purpose.

Based on the above observation, we are to identify the unnecessary scan operations from a given test sequence with sparse information and to reduce the scan operations by selectively scanning. In the discussion, we assume that the scanned circuit has separate clock lines for the scanned flip-flops and the nonscanned flipflops as shown in Figure 1. These two clock lines can be independently controlled to hold-data(disabled) or normal-operation(enabled) in each type of flip-flops. There is an additional control line in the scanned circuits, the Scan-Enable line, which enables the shift operation of the scanned flip-flops. The extra feature requires little overhead besides that required for scan design.

With these three control lines in a partial scanned circuit, the scanned flip-flops can be operated as shift-register(S), hold-data $(\mathrm{H})$, or normaloperation( $\mathrm{N})$ and the unscanned flip-flops can be in normal-operation $(\mathrm{N})$ or hold-data $(\mathrm{H})$. For example, the phase-one testing is performed in the NN mode and the shifting operation of the scan chain is in the $\mathrm{SH}$ mode.

Four cases of unnecessary scans are identified and methods of selective scan are devised to eliminate them.

1. Actual Scan Requirement: The scan is only performed to shift in all the effective test bits to the right position and to shift out all the faulteffect response bits.

For a test pattern, the straightforward (TTCs) is $S+1$, where $S$ is the number of scan elements in the scan path. However, from test generation, it is possible to obtain the response bits of $T_{i}$ to be shifted out and the effective test bits of $T_{i+1}$ to be shifted in. The positions of these bits on the scan path allow us to determine the number of actual scan requirement for $T_{i+1}$, which is less than or 


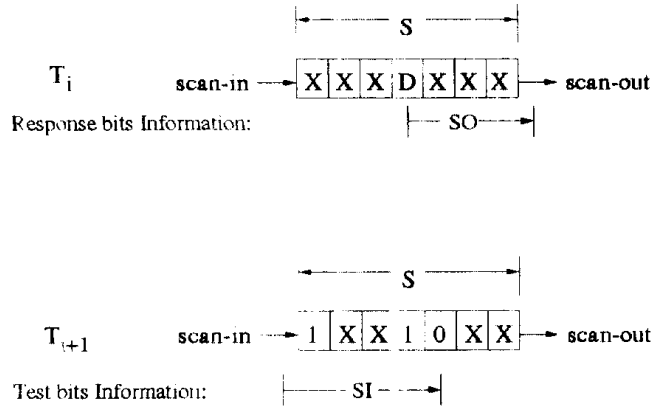

Figure 2: Actual Scan Requirement

equal to $S$. For example, in Figure 2, the number of shift of the pattern is $M A X(S O, S I)$, where $S O(S I)$ is the the maximum distance of the effective response(test) bits from the scan-out(scanin) pin.

This reduction method is supported by any partial scan designs. However, the reduction is heavily dependent on the ordering of scan elements.

2. Delayed Scan-Out: The scan-out operations are not performed unless a fault-effect bit is in danger of being overuritten by a new response.

As described earlier, the observation of fault effect on SFFs only happens occasionally. Hence additional scan reduction can be obtained if the rare fault effects can be held in the scan path so that their scan-out can be overlapped with the scan-in operations of the succeeding test patterns. For example, in Figure 2, if $S O>S I$ and there is no SFF to be observed for the succeeding patterns such as $T_{i+1}$ and $T_{i+2}$, then only $S I$ bits are shifted and the remaining fault effect bits of $T_{i}$ can be held in the scan path to overlap its shiftout with the scan-in operations of the succeeding patterns.

This reduction method requires the scanned flipflops to hold the scanning data without being over-written by the new but useless responses. This ability is supported in our scan facility.

3. Reusable Scan-In: The scan-in operations are performed only to the point that all effective test bits are obtained.

In our scan facility, a bit in the scan path can be held without being over-written by the responses. This bit can be a fault effect as in the delayed scan-out. It can also be a scan-in test bit which can then be used in the succeeding patterns at the farther positions of the scan path. For example, if there is a ' 1 ' at the third SFF of the scan path for $T_{i}$ and a single ' 1 ' is also needed at the fifth SFF for $T_{i+1}$, then only two shift operations between $T_{i}$ and $T_{i+1}$ are required instead of the original five shift-operations. Therefore the scan number can be reduced. This reduction is possible only when $T_{i}$ does not need to observe any SFF and the original pattern is kept in the scan path by the hold operation.

4. Concurrent Shift: If there is no immediate fault effect to be observed after applying a test pattern, one shifi operation of the scan path can operate concurrently with the test application of the circuit.

This concurrent shift utilizes the independen$t$ clocks of our scanned circuit, in which the scanned flip-flops perform the scan-in operation of the next pattern while the unscanned flip-flops are loading the response. The concurrent shift is able to reduce at most one shift per pattern. The reduction is in general not significant but there is one advantage in the concurrent shift. Unlike previous cases, it is independent of the ordering of scan path.

\subsection{Rearrangement of Scan Path}

The effectiveness of most selective scan methods described above depend on the ordering of SFFs in the scan path. Here we consider the ordering problem with the assumption that all permutations of SFFs are feasible. In other words, no physical constraint on the ordering is considered in this paper. However, we would like to point out that, in our reordering procedure, it is possible to take the constraints into account.

The shift requirement of controlling/observing an SFF directly depends on the position of this SFF in the scan path. An SFF placed close to the scan-out pin will require approximately $S$ shift operations to set its value but only a few shifts can scan out its value for observation. On the other hand, an SFF positioned near the scan-in pin is significantly easier for scan-in. The ordering consequently must be a tradeoff of these two conflictive demands. Our heuristic is to take as a weight the difference of a SFF's controlling frequency and observing frequency obtained from test generation. And the ordering of the SFFs from the scan-in pin to the scan-out pin is determined by the descending order of this weight.

By our experiments, this ordering of SFFs gives fairly good results for our reduction methods.

\section{Results and Discussion}

ISCAS sequential benchmark circuits [3] are used to evaluate our reduction strategy. Each circuit is first partially scanned to attain an acceptable fault coverage, then two-phase test generated, and finally selectively scanned with scan path rearrangement. The scanned flip-flops are selected based on a structural criteria[9] to achieve reasonable fault coverage. For the evaluated circuits, the performance of the selection criteria is comparable to that of $[11,12,13]$. The test pattern generator is a conventional propagate-thenjustify generator and has performance comparable to STG3[10].

The evaluation result is shown in Table 1 . In this table, "Nonscan" is the result of the original scanless 
circuits, "Scan" the partial scan circuits with "SFF" FFs scanned, "2-Ph" the two-phase test generation, and "Redu" the final reduced result. For each case, the upper datum is the fault coverage and the lower datum is the TTCs (Total Test Clock-cycles).

We can see from Table 1 that the two-phase test generation alone reduces the TTCs to $65.6 \%$ of the conventional partial scan. This reduction is achieved with ordinary scan path and without any selective scan. Applying the selective scan techniques and the ordering of SFFs, the TTCs can be reduced to $20 \%$ averagely, which indicates a very significant reduction in the testing cost.

In Table 2, we show the cumulative effect of our test reduction methods. The column " $2-\mathrm{Ph}^{\text {" is the }}$ same as Table 1 , and " $+\mathrm{X}$ " is the result when deliberately leaving don't-care on SFFs in the phase-two of test generation. Note that TCCs of " $+\mathrm{X}$ " are more than "2-Ph" because the ATPG's heuristic of setting the unassigned PIs in phase-two is sacrificed for the benefit of the later selective scan. The column "+Actual" is the actual shift requirement. And the column " $+\mathrm{IO}$ " is the result when further applying the delayedscan-out and reusable-scan-in methods, while column "+Concur" the concurrent-shift method. The average efficiency of every reduction methods can be found in the last row of Table 2. Note that the increase of TCCs in " $+\mathrm{X}$ " is more than justified when subsequent selective scan is applied and TTCs are drastically reduced. Most of our proposed methods, except concurrent shift, provide significant reduction in TTCs. Since the concurrent-shift at best can save one clockcycle for every pattern, its effectiveness is not so good as the other methods. On average, $80 \%$ total test clock-cycles reduction is achieved without restriction on the SFF ordering.

One might notice that the final fault coverage is slightly different from the original "Scan". This is because the modified ATPG sacrifice the heuristics for setting the unsigned SPIs and some faults are not detected without these heuristics. But for the circuit S5378, the fault coverage after test reduction become higher. This may due to that in the scan reduction, values are assigned to the unassigned SFFs during test generation and hence unintentionally detect some previously undetected faults

\section{Conclusion}

The reduction of test application time in scandesigned circuits had been considered in this paper. The reduction problem had been investigated from three aspects: the test generation, selective scan, and rearrangement of scan path. The two phase testing strategy had been proposed to employ scan only for the hard-to-detect faults. Four cases of selective scan had also been identified. Furthermore, an ordering heuristic without layout constraint had been proposeded to maximize the reduction of unnecessary scans and hence the test application time. Applying these reduction methods, the total test clock-cycles can be reduced to only $20 \%$ on average for ISCAS sequential benchmark circuits with partial scan.

Although the evaluation had been performed for the partial scan case with single path, we like to point out that all our discussions are also applicable to other scan designed circuits.

\section{References}

[1] E.B. Eichelberger and and T.W. Williams, "A Logic Design Structure for LSI Testability," J. Design Automa. Proc. Fault-Tolerant Comput., pp.165-178, May 1978.

[2] E. Trischler, "Incomplete Scan Path with an Automatic Test Generation Methodology," Proc. ITC, pp.153-162, Nov. 1980.

[3] F. Brglez, D. Bryan and K. Kozminski, "Combinational Profiles of Sequential Benchmark Circuits," Proc. ISCAS, pp.1929-1934, May 1989.

[4] S. Bhawmik, C.J. Lin, K.-T. Cheng and V.D. Agrawal, "PASCANT: A Partial Scan and Test Generation System," Proc. CICC, pp.17.3.117.3.4, 1991.

[5] S.P. Morley and R.A. Marlett, "Selectable Length Partial Scan: A Method to Reduce Vector Length," Proc. ITC, pp.385-392, 1991.

[6] S. Osstdijk, F. Beenker and L. Thijssen, "A Model for Test-Time Reduction of Scan Testable Circuits," Proc. ETC, pp.243-252, 1991.

[7] R. Gupta and M.A. Breuer, "Ordering Storage Elements in a Single Scan Chain," Proc. ICCAD, pp.408-411, 1991.

[8] J.-D. Pan, "Sequential Test Pattern Generation," Master Thesis, National Taiwan University, 1991.

[9] W. Lai and C. Lin, "Selection of Scan Flip-Flups in Partial Scan Design", Proc. 3rd VLSI/CAD Workshop, pp. 11-18, 1992.

[10] W. T. Cheng and S. Davidson, "Sequential Circuit Test Generation Benchmark Result" Proc. ISCAS, pp. 1931-1934, 1989

[11] K. S. Kim and C. R. Kime, "Partial Scan by Use of Empirical Testability", Proc. ICCAD, pp. 314$317,1990$.

[12] K. T. Cheng and V. D. Agrawal, "An Economical Scan Design for Sequential Logic Test Generation", Proc. FTCS, pp. 28-35, 1989.

[13] D. H. Lee and S. M. Reddy, "On Determining Scan Flp-Flops in Partial Scan Design", Proc. ICCAD, pp. 322-325, 1990 
Table 1: Results of Test Reduction

\begin{tabular}{|c|c|c|c|c|c|c|}
\hline bench & FFs & SFFs & Nonscan & Scan & 2-ph & Redu \\
\hline s208 & 8 & 3 & $\begin{array}{c}63.72 \\
124\end{array}$ & $\begin{array}{c}90.23 \\
671\end{array}$ & $\begin{array}{c}90.23 \\
631\end{array}$ & $\begin{array}{c}90.23 \\
336\end{array}$ \\
\hline s298 & 14 & 1 & $\begin{array}{c}86.04 \\
137\end{array}$ & $\begin{array}{c}94.81 \\
693\end{array}$ & $\begin{array}{c}94.81 \\
514\end{array}$ & $\begin{array}{c}94.81 \\
362\end{array}$ \\
\hline s344 & 15 & 5 & $\begin{array}{c}96.20 \\
77\end{array}$ & $\begin{array}{c}99.12 \\
551\end{array}$ & $\begin{array}{c}98.83 \\
256\end{array}$ & $\begin{array}{c}98.54 \\
129\end{array}$ \\
\hline s382 & 21 & 6 & $\begin{array}{c}76.19 \\
140\end{array}$ & $\begin{array}{l}97.49 \\
1931\end{array}$ & $\begin{array}{l}97.49 \\
1504\end{array}$ & $\begin{array}{c}97.24 \\
697\end{array}$ \\
\hline s386 & 6 & 2 & $\begin{array}{c}81.77 \\
250\end{array}$ & $\begin{array}{c}94.79 \\
626\end{array}$ & $\begin{array}{c}94.79 \\
480\end{array}$ & $\begin{array}{c}94.79 \\
369\end{array}$ \\
\hline 8400 & 21 & 7 & $\begin{array}{c}76.65 \\
226\end{array}$ & $\begin{array}{c}97.88 \\
1247\end{array}$ & $\begin{array}{c}97.88 \\
953\end{array}$ & $\begin{array}{c}97.88 \\
667\end{array}$ \\
\hline $\mathrm{s} 420$ & 16 & 7 & $\begin{array}{c}41.63 \\
142\end{array}$ & $\begin{array}{l}93.49 \\
3999\end{array}$ & $\begin{array}{l}93.49 \\
3869\end{array}$ & $\begin{array}{l}93.49 \\
1057\end{array}$ \\
\hline s444 & 21 & 6 & $\begin{array}{c}76.16 \\
167\end{array}$ & $\begin{array}{r}94.30 \\
2967\end{array}$ & $\begin{array}{l}94.30 \\
2063\end{array}$ & $\begin{array}{l}94.30 \\
1197\end{array}$ \\
\hline s510 & 6 & 4 & $\begin{array}{c}0.0 \\
0\end{array}$ & $\begin{array}{c}100.0 \\
749\end{array}$ & $\begin{array}{c}100.0 \\
749\end{array}$ & $\begin{array}{c}100.0 \\
977\end{array}$ \\
\hline 8526 & 21 & 7 & $\begin{array}{c}67.03 \\
539\end{array}$ & $\begin{array}{l}98.74 \\
7071\end{array}$ & $\begin{array}{l}98.74 \\
5202\end{array}$ & $\begin{array}{l}98.74 \\
1886\end{array}$ \\
\hline s641 & 19 & 2 & $\begin{array}{c}86.30 \\
146\end{array}$ & $\begin{array}{c}94.00 \\
668\end{array}$ & $\begin{array}{c}94.00 \\
256\end{array}$ & $\begin{array}{c}94.00 \\
211\end{array}$ \\
\hline s713 & 19 & 7 & $\begin{array}{c}81.93 \\
139\end{array}$ & $\begin{array}{c}88.12 \\
919\end{array}$ & $\begin{array}{c}88.12 \\
346\end{array}$ & $\begin{array}{c}88.12 \\
212\end{array}$ \\
\hline $\mathbf{s 8 2 0}$ & 5 & 2 & $\begin{array}{c}95.76 \\
909\end{array}$ & $\begin{array}{l}100.0 \\
1334\end{array}$ & $\begin{array}{l}100.0 \\
1073\end{array}$ & $\begin{array}{l}100.0 \\
1019\end{array}$ \\
\hline $\mathbf{s 8 3 8}$ & 32 & 16 & $\begin{array}{c}29.64 \\
109\end{array}$ & $\begin{array}{l}95.10 \\
12137\end{array}$ & $\begin{array}{l}95.10 \\
11821\end{array}$ & $\begin{array}{l}95.10 \\
3455\end{array}$ \\
\hline s953 & 29 & 3 & $\begin{array}{c}8.25 \\
10\end{array}$ & $\begin{array}{c}99.91 \\
2003\end{array}$ & $\begin{array}{l}99.91 \\
1925\end{array}$ & $\begin{array}{l}99.91 \\
1268\end{array}$ \\
\hline $\mathrm{s} 1423$ & 74 & 17 & $\begin{array}{c}67.39 \\
344\end{array}$ & $\begin{array}{l}87.20 \\
40643\end{array}$ & $\begin{array}{l}87.86 \\
13501\end{array}$ & $\begin{array}{l}86.54 \\
1338\end{array}$ \\
\hline 85378 & 179 & 32 & $\begin{array}{c}70.43 \\
261 \\
\end{array}$ & $\begin{array}{r}93.31 \\
37091 \\
\end{array}$ & $\begin{array}{l}93.33 \\
30521 \\
\end{array}$ & $\begin{array}{l}93.35 \\
7207 \\
\end{array}$ \\
\hline average & $\begin{array}{l}\text { Fcov. } \\
\text { TTCs }\end{array}$ & $31 \%$ & $\begin{array}{c}65.005 \\
219\end{array}$ & $\begin{array}{c}95.205 \\
6782\end{array}$ & $\begin{array}{c}95.23 \\
4451\end{array}$ & $\begin{array}{l}95.12 \\
1317\end{array}$ \\
\hline ratio & & & & 100 & 65.6 & 19.4 \\
\hline
\end{tabular}

Table 2: Accumulative Effect of Reduction Techniques

\begin{tabular}{|c|c|c|c|c|c|c|}
\hline bench & Scan & 2-ph & $+X$ & +Actual & +10 & + Concur \\
\hline \multirow[t]{2}{*}{$\mathrm{s} 208$} & 90.23 & 90.23 & 90.23 & 90.23 & 90.23 & 90.23 \\
\hline & 671 & 631 & 931 & 399 & 369 & 336 \\
\hline s298 & $\begin{array}{c}94.81 \\
693\end{array}$ & $\begin{array}{c}94.81 \\
514\end{array}$ & $\begin{array}{c}94.81 \\
582\end{array}$ & $\begin{array}{c}94.81 \\
570\end{array}$ & $\begin{array}{c}94.81 \\
438\end{array}$ & $\begin{array}{c}94.81 \\
362\end{array}$ \\
\hline s344 & $\begin{array}{c}99.12 \\
551\end{array}$ & $\begin{array}{c}98.83 \\
256\end{array}$ & $\begin{array}{c}98.54 \\
322\end{array}$ & $\begin{array}{c}98.54 \\
200\end{array}$ & $\begin{array}{c}98.54 \\
142\end{array}$ & $\begin{array}{c}98.54 \\
\end{array}$ \\
\hline \multirow{2}{*}{ s382 } & 97.49 & 97.49 & 97.24 & 97.24 & 93.24 & 97.24 \\
\hline & 1931 & 1504 & 3044 & 1390 & 846 & 697 \\
\hline s386 & 94.79 & 94.79 & 94.79 & 94.79 & 94.79 & 94.79 \\
\hline 8400 & $\begin{array}{c}626 \\
97.88 \\
1247\end{array}$ & $\begin{array}{c}480 \\
97.88 \\
953\end{array}$ & $\begin{array}{c}519 \\
97.88 \\
1729\end{array}$ & $\begin{array}{c}506 \\
97.88 \\
1187\end{array}$ & $\begin{array}{c}416 \\
97.88 \\
744\end{array}$ & $\begin{array}{c}369 \\
97.88 \\
667\end{array}$ \\
\hline \multirow[t]{2}{*}{8420} & 93.49 & 93.49 & 93.49 & 93.49 & 93.49 & 93.49 \\
\hline & 3999 & 3869 & 5533 & 1978 & 1326 & 1057 \\
\hline \multirow[t]{2}{*}{ s444 } & 94.30 & 94.30 & 94.30 & 94.30 & 94.30 & 94.30 \\
\hline & 2967 & 2063 & 6193 & 2482 & 1360 & 1197 \\
\hline \multirow[t]{2}{*}{ s510 } & 100.0 & 100.0 & 100.0 & 100.0 & 100.0 & 100.0 \\
\hline & 749 & 749 & 1474 & 1424 & 1.160 & 977 \\
\hline \multirow[t]{2}{*}{8526} & 98.74 & 98.74 & 98.74 & 98.74 & 98.74 & 98.74 \\
\hline & 7071 & 5202 & 7898 & 3572 & 2244 & 1886 \\
\hline \multirow[t]{2}{*}{8641} & 94.00 & 94.00 & 94.00 & 94.00 & 94.00 & 94.00 \\
\hline & 668 & 256 & 325 & 272 & 223 & 211 \\
\hline \multirow[t]{2}{*}{ s713 } & 88.12 & 88.12 & 88.12 & 88.12 & 88.12 & 88.12 \\
\hline & 919 & 346 & 402 & 281 & 225 & 212 \\
\hline \multirow[t]{2}{*}{ s820 } & 100.0 & 100.0 & 100.0 & 100.0 & 100.0 & 100.0 \\
\hline & 1334 & 1073 & 1139 & 1127 & 1075 & 1019 \\
\hline \multirow[t]{2}{*}{ s838 } & 95.10 & 95.10 & 94.75 & 94.75 & 95.10 & 95.10 \\
\hline & 12137 & 11821 & 24809 & 12616 & 3944 & 3455 \\
\hline \multirow[t]{2}{*}{ s953 } & 99.91 & 99.91 & 99.91 & 99.91 & 99.91 & 99.91 \\
\hline & 2003 & 1925 & 2665 & 2557 & 1676 & 1268 \\
\hline \multirow[t]{2}{*}{ s1423 } & 87.20 & 87.86 & 78.09 & 78.09 & 85.08 & 86.54 \\
\hline & 40643 & 13501 & 12727 & 2165 & 1387 & 1338 \\
\hline \multirow[t]{2}{*}{ s5378 } & 93.31 & 93.33 & 93.31 & 93.31 & 93.35 & 93.35 \\
\hline & 37091 & 30521 & 44942 & 17863 & 7786 & 7207 \\
\hline \multirow[t]{2}{*}{ average } & 95.205 & 95.23 & 94.60 & 94.60 & 94.80 & 95.12 \\
\hline & 6782 & 4451 & 6778 & 2976 & 1492 & 1317 \\
\hline ratio & 100 & 65.6 & 99.9 & 43.9 & 22 & 19.4 \\
\hline
\end{tabular}

